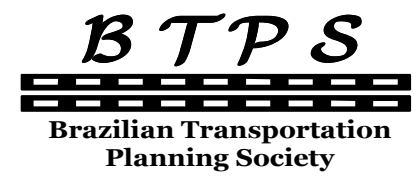
Journal of Transport Literature
Vol. 7, n. 2, pp. 207-234, Apr. 2013
Research Directory

JTL|RELIT

www.transport-literature.org ISSN 2238-1031

\title{
Modelo de quatro etapas aplicado ao planejamento de transporte de carga
}

\author{
[Four-step model applied for freight transportation planning] \\ Cristiane Duarte Ribeiro de Souza*, Márcio de Almeida D'Agosto \\ Universidade Federal do Rio de Janeiro (UFRJ), Brazil
}

Submitted 2 May 2012; received in revised form 2 Jul 2012; accepted 11 Jul 2012

\begin{abstract}
Resumo
Este artigo tem como objetivo analisar estudos de planejamento de transporte de carga que utilizam o modelo de quatro etapas, ferramenta capaz de estimar o fluxo de tráfego interzonal, considerando a geração e distribuição de viagens, a escolha modal e a alocação de fluxo, destacando sua abrangência e utilidade em apoiar o desenvolvimento socioeconômico do país. Foi realizada revisão bibliográfica em periódicos indexados, teses, dissertações e relatórios técnicos, selecionando-se 44 trabalhos com destacando para 7 trabalhos que enfocam o modelo de quatro etapas integralmente. Verificou-se que este modelo é versátil, pode ser empregado de forma integral ou parcial, considerando fluxos de unidade de massa ou de veículos, no caso destes últimos diretamente aplicáveis ao dimensionamento de infraestrutura de tráfego, em todos os níveis de abrangência geográfica (de municípios a países), sem limitação do tipo de carga que pode ser transportada por qualquer dos cinco modos de transporte ou suas combinações, configurando ferramenta de planejamento estratégico e apoio ao desenvolvimento socioeconômico do país.
\end{abstract}

Palavras-Chave: planejamento, transporte de carga, modelo de quatro etapas.

\begin{abstract}
This paper aims to analyze existing studies on freight transport planning that use the four step model, a tool that estimates inter-zonal traffic flows considering trip generation and distribution, modal choice and flow allocation; highlighting its comprehensiveness and usefulness in supporting the country's socioeconomic development. A literature review was performed in indexed journals, theses, dissertations and technical reports, selecting 44 papers and highlighting 7 papers that fully focus on the four step model. It was verified that this model is versatile and can be used in full or partially; considering mass or vehicle flows, the latter being directly applicable to the design of traffic infrastructure; at all levels of geographical coverage (from municipalities to countries); and without limitations on the type of freight that can be transported by any of the five modes of transport or their combinations. Therefore, this model is a strategic planning tool and a support for the socioeconomic development of the country.
\end{abstract}

Key words: planning, freight transportation, four-step model.

*Email: cristiane@ltc.coppe.ufrj.br.

\section{Recommended Citation}

Souza, C. D. R. and D'Agosto, M. A. (2013) Modelo de quatro etapas aplicado ao planejamento de transporte de carga. Journal of Transport Literature, vol. 7, n. 2, pp. 207-234.

- JTL/RELIT is a fully electronic, peer-reviewed, open access, international journal focused on emerging transport markets and published by BPTS - Brazilian Transport Planning Society. Website www.transport-literature.org. ISSN 2238-1031. 


\section{Introdução}

O transporte é primordial para o desenvolvimento econômico de um país. De acordo com Crainic e Laporte (1997), é ele que dá suporte e torna possível a maior parte das atividades sociais e econômicas. $\mathrm{O}$ transporte de carga, em particular, tem sua importância associada não apenas a participação na composição do produto interno bruto do país, mas também pela crescente influência que a transferência, coleta e distribuição de carga têm no desempenho dos segmentos econômicos produtivos e no bem estar da sociedade.

Um adequado planejamento é a chave para se alcançar a efetividade do setor de transporte de carga e para uma abordagem estratégica este requer uma análise abrangente dos aspectos econômicos, sociais e ambientais da região em estudo. Complementarmente, considerado o papel do transporte como função logística, outros aspectos devem se considerados, tais como: configuração da rede, localização de instalações (armazéns e centros de distribuição), escolha modal (combinação dos modos de transporte que realizam a operação), dimensionamento das frotas, políticas de estoque e sistema de comunicação e controle.

Em sua aplicação tradicional, o planejamento de transporte de carga utiliza o modelo de análise previsão de demanda de quatro etapas com finalidade de estimar os fluxos futuros de carga na região em estudo. É baseado neste resultado que os tomadores de decisão elaboram e avaliam diferentes alternativas para implantação ou aprimoramento da efetividade do sistema de transportes existente.

Para o Brasil, que enfrenta crônicos problemas quanto ao transporte de carga associados à dinamização do crescimento econômico e melhoria da qualidade de vida da sociedade, a geração e divulgação de conhecimento científico sobre planejamento de transporte de carga e o potencial de utilização do modelo de quatro etapas deveria ser incentivado. A despeito disso, em pesquisa realizada na base de periódicos da CAPES (Coordenação de Pessoal de Nível Superior), principal fonte brasileira de literatura científica, no período de 2000 a 2010 , não se encontrou publicações brasileiras relacionadas ao tema, identificando-se assim uma lacuna na geração e divulgação de conhecimento em gestão de transporte de carga. 
De forma a suprimir esta lacuna, o presente trabalho tem por objetivo analisar uma seleção de estudos de planejamento de transporte de carga, identificados a partir de revisão bibliográfica realizada conforme Figura 4 (item 3), que utilizam o modelo de quatro etapas como modelo de análise e previsão de demanda, verificando como este modelo poderia ser aplicado ao planejamento de transporte de carga no Brasil, de modo a apoiar o desenvolvimento socioeconômico do país. Para tanto, os estudos foram analisados quanto ao tipo de fluxo (se consideram os fluxos em unidade de massa ou volume de veículos), a abrangência geográfica (urbano, interurbano, estadual e nacional), variáveis consideradas na modelagem, modos de transporte considerados, etapas do modelo consideradas, produtos considerados e local do estudo.

Verificou-se que o modelo de quatro etapas é uma ferramenta versátil para aplicação a análise e previsão de demanda de fluxos de carga, que pode ser empregado de forma integral ou parcial, considerando fluxos de unidade de massa ou de veículos, no caso destes últimos diretamente aplicáveis ao dimensionamento de infraestrutura de tráfego, em todos os níveis de abrangência geográfica (de cidades a países), para um abrangente conjunto de cargas (de carga geral de alto valor agregado a granéis minerais) que podem ser transportadas por todos os cinco modos de transporte ou suas combinações.

Por outro lado, o modelo de quatro etapas quando aplicado a análise e previsão de demanda de fluxos de carga não parece ser capaz de modelar diretamente os aspectos relacionados ao planejamento estratégico do transporte de carga como função logística, representando uma aparente limitação da técnica a ser ultrapassada.

Além desta introdução, este artigo está dividido em mais cinco itens. O segundo conceitua o processo de planejamento de transportes de carga, o terceiro descreve o modelo de quatro etapas. No item 4 apresenta-se a revisão bibliográfica e a síntese dos estudos que enfocam a aplicação do modelo de quatro etapas. A discussão da revisão bibliográfica é apresentada no item 5. Por fim apresentam-se as considerações finais, limitações e sugestões de trabalhos futuros. 


\section{Planejamento de transporte de carga}

O planejamento de transporte busca antecipar ou adequar as necessidades de transporte de uma região ao seu desenvolvimento socioeconômico (Adler, 1978). De acordo com Marra (1999), o adequado planejamento do transporte de carga pode resultar em efeitos positivos à efetividade do sistema de transporte como um todo, contribuindo para uma menor interferência da movimentação de carga na rede de tráfego de passageiros, para a redução do custo total de transporte e, por consequência, do custo final da carga transportada.

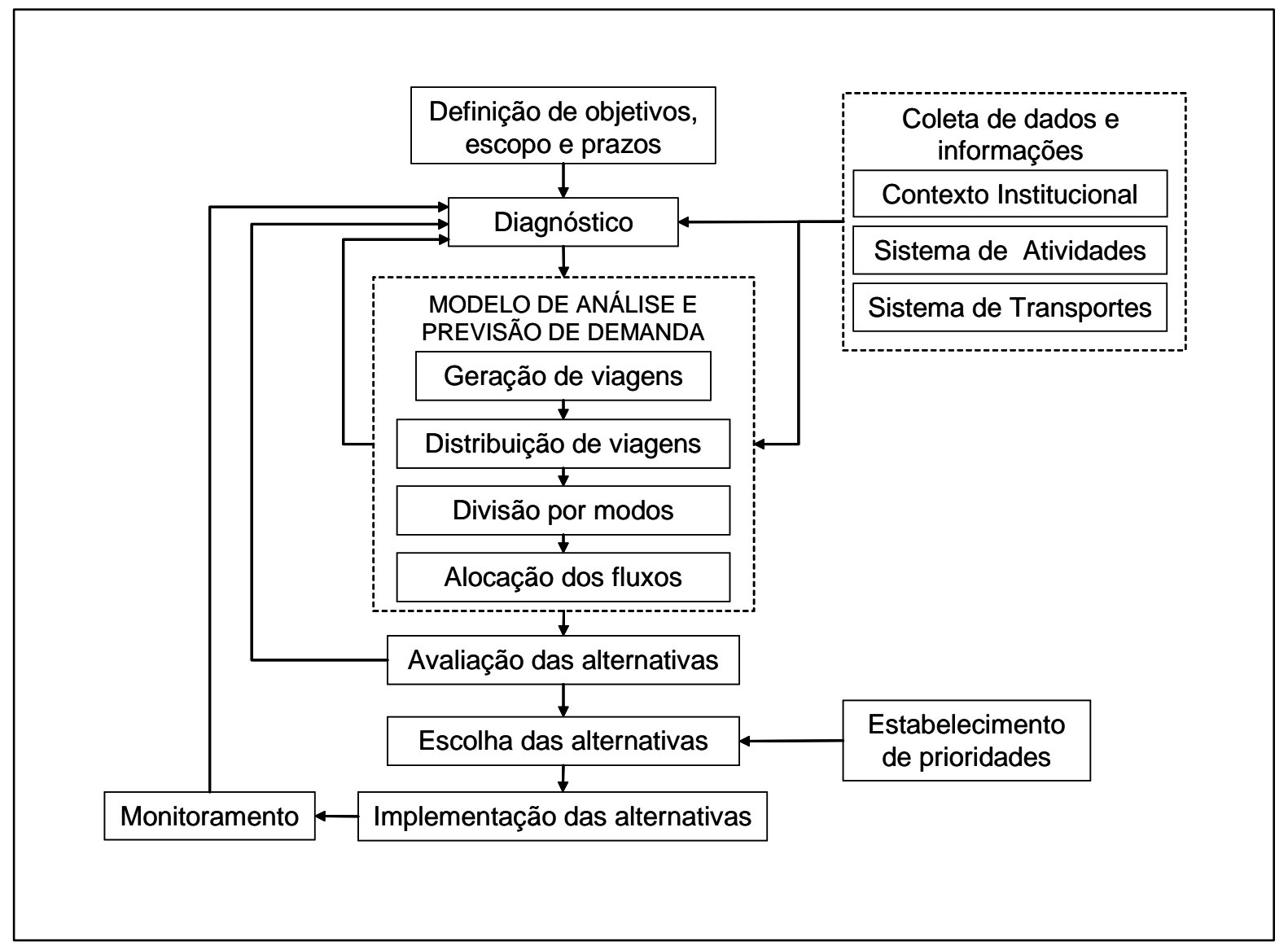

Figura 1 - Processo de planejamento estratégico tradicionalmente utilizado ${ }^{1}$

Pode-se dividir o planejamento de transporte em um processo sequencial conforme apresenta a Figura 1. Este processo envolve a definição de objetivos, escopo e prazos, a coleta de dados e informações referentes ao contexto institucional, ao sistema de atividades e ao sistema de transporte existente. De posse dessas informações, é realizado o diagnóstico da situação atual.

\footnotetext{
${ }^{1}$ Fonte: elaboração própria partir de Adler, 1978, Anderson, 1994, FHA, 1999, MT, 2007.
} 
Na parte central do processo encontra-se o modelo de análise e previsão de demanda, sendo que o mais utilizado neste caso é o modelo de quatro etapas. O próximo passo é a avaliação das alternativas resultantes do modelo de quatro etapas, que devem ser escolhidas de acordo com as prioridades estabelecidas. Por último, deve-se implementar as alternativas escolhidas. Para maior eficácia, o planejamento deve ser monitorado periodicamente, buscando incorporar as transformações que ocorreram no período posterior a sua elaboração.

\section{Modelo de quatro etapas}

O modelo de análise e previsão de demanda de quatro etapas é tradicionalmente empregado pelo setor de transportes. O entendimento de sua base conceitual baseou-se em Menezes (1971), Morlok (1978), Tedesco (2008), Magalhães (2008), Odgen (1992) e Marra (2009).

A finalidade do modelo de quatro etapas é prever a demanda de tráfego, por meio da análise e da determinação da quantidade e do tipo de viagens entre zonas em uma determinada região por meio da aplicação sequencial das etapas de geração de viagens, distribuição de viagens, divisão modal e alocação de tráfego. Para a realização de cada uma dessas etapas pode-se utilizar métodos qualitativos ou quantitativos (Ballou, 2001), sendo o primeiro baseado em julgamento, intuição, pesquisas ou técnicas comparativas e o segundo em modelos matemáticos baseados na análise de dados passados que podem ser divididos em dois grupos: (1) métodos baseados em séries temporais e (2) métodos causais (Marins, 2011).

Os métodos baseados em séries temporais fundamentam-se em observações passadas para prever o comportamento futuro, sendo possível obter previsões acuradas se o futuro apresentar comportamento similar ao do passado (Makridakis et al, 1998). Como exemplo deste pode-se citar: os métodos de Média Móvel Simples (MMS), Projeção de Tendência (PT), modelos de Decomposição Aditiva (DA) e Multiplicativa (DM).

Já os métodos causais baseiam-se na previsão de outra variável que possua relação com o produto. Como exemplo de técnicas utilizadas por esses métodos têm-se as taxas de crescimento, regressões simples e regressões múltiplas (Marins, 2011). 
A geração de viagens tem o objetivo de estimar a produção e a atração de cargas (unidade de massa ou viagens de veículos de carga) para cada uma das zonas de transporte (ZT) da área de estudo tendo como base um período de tempo (dia, semana, mês, ano) típico da situação futura.

Neste caso, é comum o uso de métodos causais, na forma de taxas de crescimento ou regressão (linear ou não), onde a geração de viagens é função do uso do solo (industrial, comercial, residencial) e de aspectos sócio-econômicos (empregos, renda, número de residências, número de estabelecimentos industriais e comerciais, assim como sua capacidade). Quando aplicado ao transporte de carga este modelo exige a tipificação da carga movimentada, o que pode ser difícil e trabalhoso se houver muita diversificação em função dos setores de atividade e das necessidades dos usuários finais e dos mercados onde atuam.

A distribuição de viagens tem por objetivo estimar o número de viagens $\left(T_{i j}\right)$ entre as ZT (i origens e $\mathrm{j}$ destinos) criando uma matriz de origem e destino (O/D) com base nos resultados obtidos na etapa de geração de viagens. Nesta etapa pode-se utilizar o método de fator de crescimento, fator sócio-econômico, custo generalizado ou modelo gravitacional (Pereira, 2007), sendo comum o uso do modelo gravitacional, vinculado ou não, conforme Equação (1), de modo que a atração e produção podem ser representadas pela quantidade de carga, pelo número de viagens de veículos ou ainda, por valor monetário da carga. Como fator de fricção utiliza-se, predominantemente, a distância entre as zonas de tráfego, podendo-se ainda utilizar o tempo de viagem ou o cuto.

$$
T_{i j}=K_{i} \cdot L_{j} \cdot \frac{P_{i} \cdot A_{j}}{d_{i j}^{a}}
$$

Onde:

Tij: fluxo de carga entre a ZT de origem i e de destino j,

Ki e Lj: fatores de ajuste,

Pi: produção de carga a ZT de origem i,

Aj: atração de carga para a ZT de destino j,

dij: fator de fricção,

a: constante de calibração 
Ainda para estabelecer a distribuição de viagens é possível utilizar a programação matemática na forma do problema de transporte. Porém, neste caso, pode ser difícil considerar as como as características específicas de geração de viagem de cada par origem-destino impactam nos fluxos interzonais, uma vez que o problema de transporte busca a composição de fluxos que minimiza o custo total da rede.

A divisão modal é necessária sempre que houver disponibilidade de mais de um modo de transporte para ligar duas ZT. No caso do transporte de carga urbana, onde predomina o transporte rodoviário, é possível abstrair que diferentes tipos e tamanhos de veículos representem diferentes modos. A escolha se baseia na avaliação de atributos característicos de cada modo, essencialmente representativos do custo, tempo de viagem (porta a porta) e confiabilidade, sendo estes relativamente importantes para os elementos envolvidos. A consideração destes atributos pode ser individual ou de forma agregada (custo generalizado).

Em função das características específicas do modelo de quatro etapas, outros fatores, como capacidade, frequência e segurança são, usualmente, considerados na etapa de alocação de fluxo. É possível que esta prática possa ser modificada quando as etapas divisão modal e alocação de fluxos forem realizadas simultaneamente, porém, isso não foi observado na literatura pesquisada.

Segundo Pereira (2007), os modelos de divisão modal podem ser determinísticos, como por exemplo, regressão linear, curvas de desvio e classificação cruzada, ou probabilísticos, como por exemplo, LOGIT binomial e multinomial. A alocação de fluxos procura avaliar a distribuição de viagens na rede de transportes existentes ou a ser criada. A escolha das rotas pode estar associada a um conjunto de fatores variando inclusive em função do equipamento a ser usado, porém, alguns fatores de decisão como rapidez, retidão, pouco congestionamento e menor caminho são comuns.

Os métodos mais comumente utilizados na etapa de alocação de fluxos são: método tudo ou nada, método STOCH, método incremental, método de restrição de capacidade, método de equilíbrio do usuário, método de equilíbrio do usuário estocástico e método do sistema ótimo (Pereira, 2007). De acordo com Marra (1999) e Ogden (1992), no caso de sistemas de transporte urbano de carga, os modelos mais utilizados para previsão de demanda podem ser baseados em deslocamento de carga ou baseados em viagens de veículos. 


\subsection{Modelos baseados em viagens}

Os modelos baseados em viagens têm seu foco no veículo (Figura 2) e pressupõem que a seleção do modo de transporte já foi realizada e, portanto, não se faz necessário efetuar a etapa de divisão modal. De acordo Ogden (1992) e Holguín-Veras e Thorson (2000), esses modelos apresentam algumas vantagens. Como exemplo, os dados de tráfego são relativamente fáceis de obter. Além disso, um crescente número aplicativos em Sistemas Inteligentes de Transporte (ITS) é capaz de seguir os movimentos dos veículos, promovendo importante fonte de dados de tráfego. Outra vantagem é que ele trata das viagens vazias de retorno que já são consideradas no modelo.



Figura 2 - Modelo de quatro etapas adaptado para carga

- abordagem com base em viagens ${ }^{2}$.

A etapa de diagnóstico fornece as informações do ano-base, desagregadas por modo de transporte $(\mathrm{m})$ necessárias para alimentar o modelo. Para obter os dados de atração (Ami) e produção $\left(\mathrm{Pm}_{\mathrm{i}}\right)$ de viagens resultantes da etapa geração de viagens, é necessário definir as taxas de geração de viagens ou o modelo de regressão específico para cada zona por modo de transporte. O resultado (Ami,Pmj) desta etapa alimenta a distribuição de viagens, que tem por objetivo distribuir as produções e atrações entre as zonas de tráfego de modo a obter o fluxo de viagens (Tmij) e a matriz origem-destino para cada modo $(\mathrm{m})$ de transporte. Usualmente

\footnotetext{
${ }^{2}$ Fonte: elaboração própria a partir de Holguín-Veras e Thorson (2000).
} 
utiliza-se o modelo gravitacional para a distribuição das viagens, conforme apresentado na introdução do item 2. A última etapa trata da alocação de tráfego, que aloca à cada segmento (y) da rede de transporte os fluxos (Tmyij) verificados na matriz origem-destino. Para melhor eficiência do modelo é necessário seu constante monitoramento.

\subsection{Modelos baseados em volume de carga}

Os modelos baseados em volume de carga, como o apresentado na Figura 3, têm o foco na quantidade de carga transportada medida em qualquer unidade de massa ou volume. Esse modelo permite captar com mais precisão os mecanismos fundamentais que direcionam a movimentação das cargas, que são determinados por seus atributos, como forma e peso por unidade.

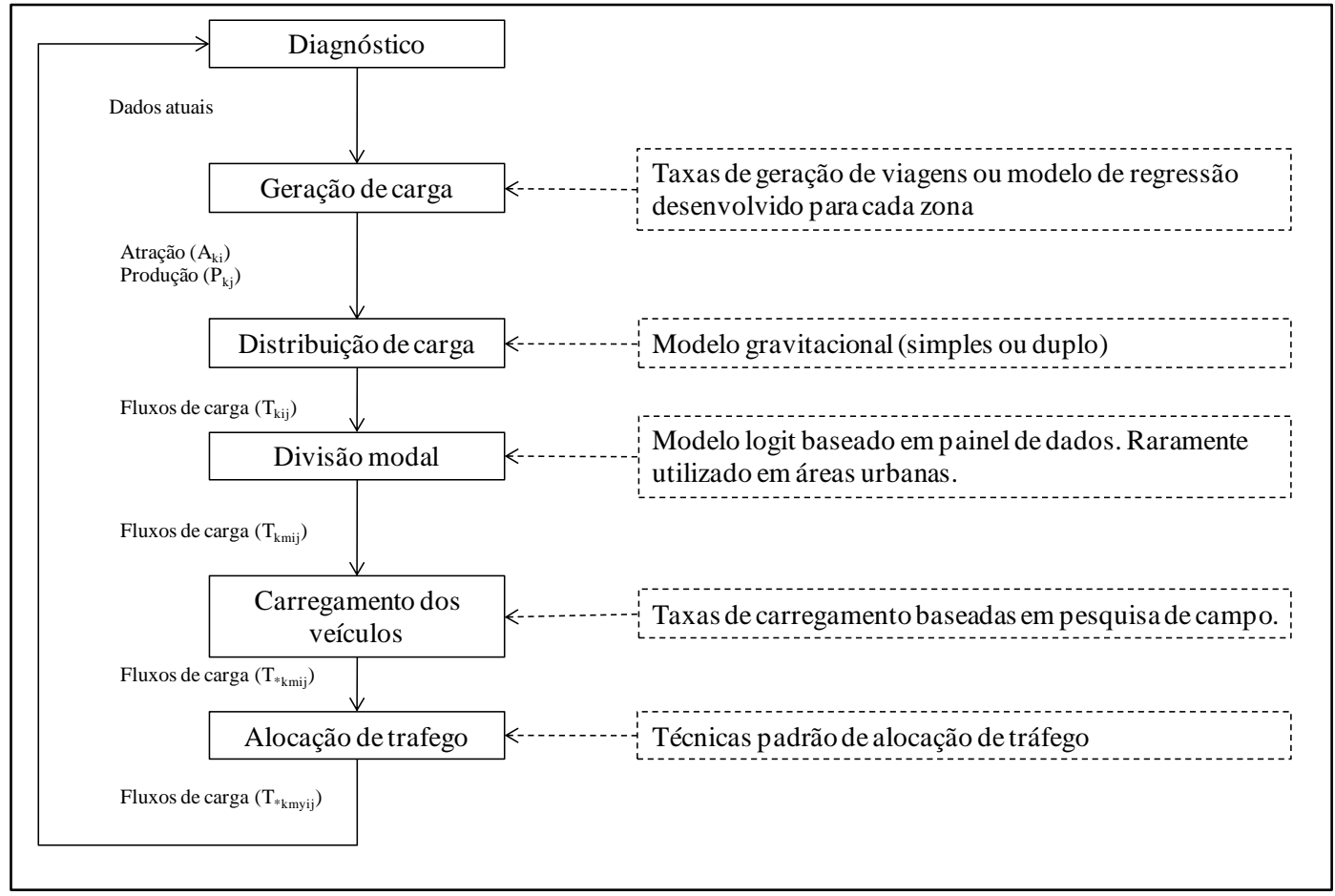

Figura 3 - Modelo de quatro etapas adaptado para carga - abordagem com base em volume de carga ${ }^{3}$.

\footnotetext{
${ }^{3}$ Fonte: elaboração própria a partir de Holguín-Veras e Thorson (2000).
} 
A etapa de diagnóstico fornece as informações do ano-base necessárias para alimentar o modelo. Para obter os dados de atração (Aki) e produção (Pki) de viagens resultantes da etapa de geração de carga, é necessário definir as taxas de geração de carga ou o modelo de regressão específico para cada zona, desagregado por tipo de carga (k). Conforme acontece com o modelo baseado em viagens, o resultado desta etapa alimenta a distribuição de carga, que tem por objetivo distribuir as produções e atrações entre as zonas de tráfego de modo a obter o fluxo de carga (Tkij) e a matriz origem-destino para cada carga.

A próxima etapa trata da divisão modal, que distribui o volume de carga obtido na matriz origem-destino entre os modos de transporte contemplados no estudo, obtendo o fluxo de carga entre zonas, por tipo de carga e por modo de transporte (Tkmij). Após a divisão modal, é necessário converter os volumes de carga em viagens de veículos ( $\mathrm{T}^{*} \mathrm{kmij}$ ), de acordo com a densidade da carga e a capacidade do veículo. A última etapa trata da alocação de tráfego (T*kmyij), que aloca os veículos obtidos na etapa anterior à rede de transporte. Para a verificação das viagens vazias são utilizados modelos complementares. Aqui também, para melhor eficiência do modelo é necessário um constante monitoramento.

\section{Revisão bibliográfica}

A revisão bibliográfica foi realizada conforme Figura 4. Assim, realizou-se uma revisão bibliográfica não exaustiva, com abrangência nacional e internacional, que considerou trabalhos voltados para as áreas de planejamento de transporte e logística, publicados a partir de 1995 (horizonte de 15 anos para o ano base de 2010), incluindo-se trabalhos anteriores sempre que o conteúdo justificasse.

Como fonte de pesquisa utilizou-se periódicos indexados, congressos, relatórios técnicos e teses e dissertações. Inicialmente, uma revisão bibliográfica preliminar foi realizada com o objetivo de identificar autores e conceitos relacionados ao tema, verificando as particularidades existentes nos modelos de planejamento aplicados ao transporte de carga.

Posteriormente, foram selecionados 44 trabalhos, 9\% nacionais e 91\% internacionais, que apresentam o enfoque do planejamento de transporte de carga, sendo 62\% artigos provenientes de periódicos e congressos, $36 \%$ relatórios técnicos e $2 \%$ teses e dissertações. 
Destes, 16 tratam do planejamento do transporte de carga de uma forma abrangente considerando o enfoque apresentado na Figura 1 e 7 apresentam uma aplicação do modelo de análise e previsão de demanda em suas quatro etapas. A determinação de modelos de geração de viagens é enfatizada em 9 trabalhos, 7 tratam prioritariamente de distribuição de viagens e 5 tem foco na escolha modal.

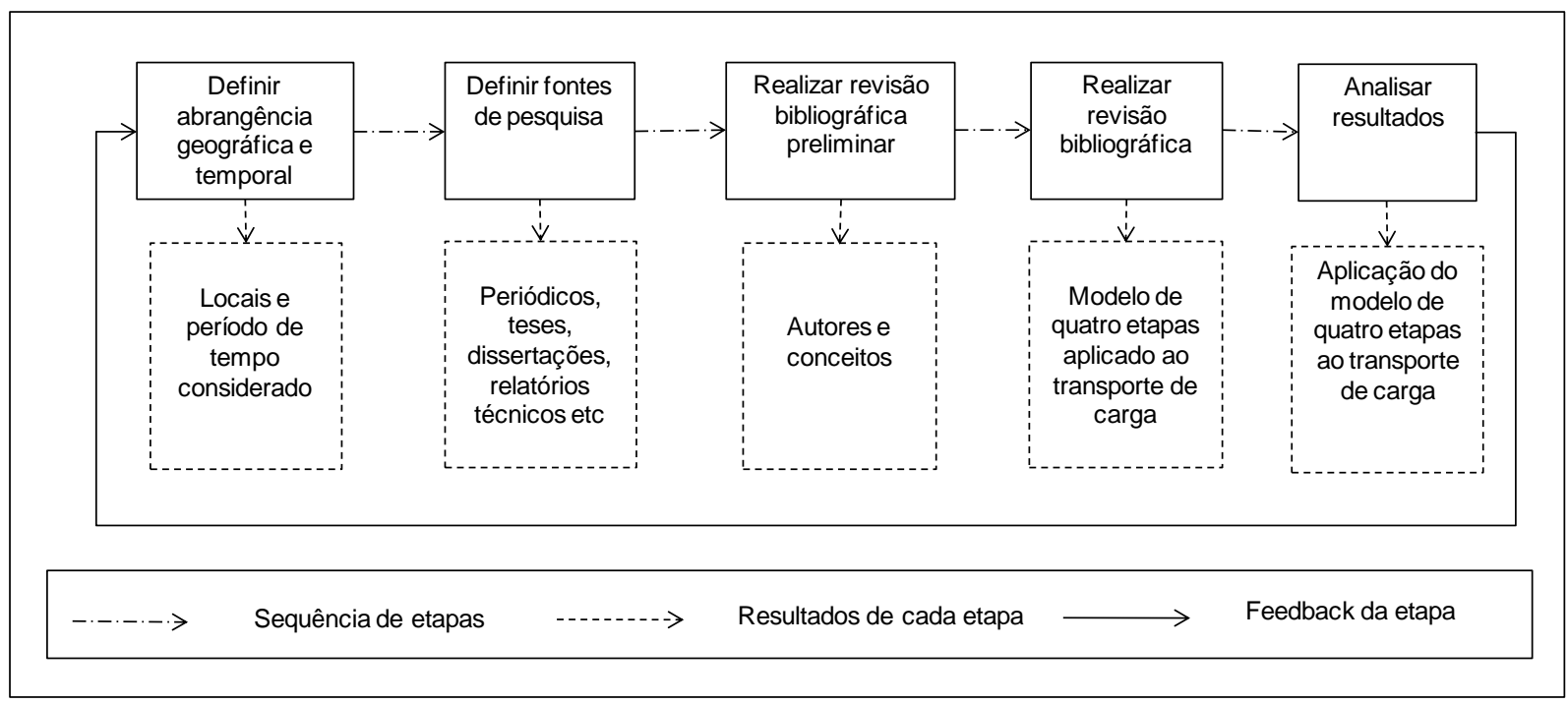

Figura 4 - Metodologia adotada para a realização da revisão bibliográfica ${ }^{4}$

Os trabalhos de Abdelwahab (1998), Garrido e Mahmassani (1999), Broga et al. (2001), Allen (2002), Kanashiro (2003), Kenneth (2003), Perry (2003), Jackson (2004), Kawamura et al. (2005), CEPA (2007), Ichihara (2007) e Miller et al. (2008) adotam uma visão abrangem para o planejamento estratégico do transporte de carga, considerando, em maior ou menor grau, aspectos relacionados ao transporte e seu impacto no desenvolvimento socioeconômico. Estes trabalhos propõem reflexões sobre a modelagem a ser adotada no caso do planejamento do transporte de carga e o enfoque utilizado é aderente ao apresentado na Figura 1, onde o modelo de análise e previsão de demanda de quatro etapas é considerado como um elemento central do processo de planejamento.

Um enfoque semelhante aos dos trabalhos relacionados anteriormente é encontrado em SAMPLAN (2001), SPC (2002), Villalobos et al. (2006) e Kveiborg e Fosgerau (2006) com maior profundidade de análise na configuração dos elementos que compõem o sistema de atividades do transporte de carga e como estes se posicionam estrategicamente quanto ao

\footnotetext{
${ }^{4}$ Fonte: elaboração própria.
} 
planejamento.

Os 16 trabalhos que apresentam o planejamento do transporte de carga de forma abrangente apresentam conteúdo que ajuda a entender como o modelo de análise e previsão de demanda de quatro etapas pode ser inserido no contexto do planejamento estratégico do transporte de carga, indicando que componentes do sistema de atividades, do sistema de transporte e do contexto institucional devem ser considerados e priorizados na atividade de planejar transportes (Figura 1). Também colaboram com a indicação sobre informações e dados que devem ser coletados e a seqüência de aplicação do procedimento de modelagem. Por posicionarem o modelo de análise e previsão de demanda no centro do modelo de planejamento de transportes, estes trabalhos também apresentam os relacionamentos entre o modelo de análise e previsão de demanda de quatro etapas e as demais atividades relacionadas ao planejamento estratégico do transporte de carga.

Souleyrette et al. (1998), Black (1999), CETU (1999), Sorratini e Smith Jr. (2000), HolguínVeras e Thorso (2001), MT (2007) e Muñuzuri et al. (2009) enfocam na aplicação do modelo de análise e previsão de demanda de quatro etapas.

Embora seja uma tarefa difícil isolar algum tipo de abordagem no campo do planejamento de transportes, os trabalhos de Harker (1985), D’Este (1996), Marra (1999), Beuthe et al (2001), Regan (2002), Combes e Leurent (2007), Macharis (2004), Ricci e Black (2005) e Janic (2007) parecem estar voltados para a determinação de modelos de geração (atração e produção) de viagens. Ogden (1977), Al-Deek et al. (2002), Celik e Guldmann (2002), ANTT (2004), Jong et al. (2004), Tavasszy et al. (1997) e Patterson et al. (2008) enfocam na modelagem da distribuição de viagens. A escolha modal é o foco dos trabalhos de Jiang et al. (1999), Arnold et al. (2004), Malchow e Kanafani (2004), Oliveira e Cury (2004) e Caris et al. (2008).

Com o intuito ilustrar a aplicação do modelo de quatro etapas para a previsão de demanda de transporte de carga, complementando a exposição da base conceitual já apresentada, realizouse a seleção de 7 trabalhos, conforme Tabela 1, que serão analisados. 
Tabela 1 - Síntese das referências selecionadas sobre aplicação do modelo de quatro etapas

\begin{tabular}{|c|c|c|c|c|c|c|c|c|c|}
\hline Referência & $\begin{array}{c}\mathrm{Nac} / \\
\text { Intl }\end{array}$ & $\begin{array}{l}\text { Tipo de } \\
\text { fluxo }\end{array}$ & $\begin{array}{c}\text { Abrangência } \\
\text { geográfica }\end{array}$ & Objetivos & $\begin{array}{c}\text { Variáveis } \\
\text { consideradas }\end{array}$ & $\begin{array}{c}\text { Modos } \\
\text { considerados }\end{array}$ & $\begin{array}{c}\text { Etapas } \\
\text { consideradas }\end{array}$ & Produtos considerados & $\begin{array}{l}\text { Local do } \\
\text { estudo }\end{array}$ \\
\hline $\begin{array}{l}\text { Munuzuri } \\
\text { et al, } 2009\end{array}$ & I & $\begin{array}{c}\text { Modelo } \\
\text { baseado } \\
\text { em VV }\end{array}$ & Urbano & $\begin{array}{l}\text { Elaborar um } \\
\text { modelo de } \\
\text { planejamento } \\
\text { simplificado }\end{array}$ & $\begin{array}{c}\text { Número de } \\
\text { atacadistas e } \\
\text { distribuidores, } \\
\text { POP }\end{array}$ & Rodoviário & $\mathrm{GV}, \mathrm{DV}, \mathrm{AT}$ & Não especificado & $\begin{array}{l}\text { Cidade de } \\
\text { Sevilla }\end{array}$ \\
\hline MT, 2007 & $\mathrm{~N}$ & $\begin{array}{c}\text { Modelo } \\
\text { baseado } \\
\text { em VC }\end{array}$ & Nacional & $\begin{array}{l}\text { Retomada do } \\
\text { processo de } \\
\text { planejamento } \\
\text { do setor de } \\
\text { transporte }\end{array}$ & $\begin{array}{c}\text { Área, PIB } \\
\text { total e per } \\
\text { capita, POP }\end{array}$ & $\begin{array}{c}\text { Aéreo, } \\
\text { Ferroviário, } \\
\text { Portuário, } \\
\text { Hidroviário e } \\
\text { Rodoviário }\end{array}$ & $\begin{array}{c}\text { GV, DV, } \\
\text { AT }\end{array}$ & $\begin{array}{l}\text { Minérios, siderúrgicos, } \\
\text { complexo de soja, cana- } \\
\text { de-açucar, fertilizantes, } \\
\text { madeira / papel/ celulose, } \\
\text { veículos, combustíveis } \\
\text { líquidos, cimento, carnes, } \\
\text { contêineres e carga geral. }\end{array}$ & Brasil \\
\hline $\begin{array}{c}\text { Sorratini e } \\
\text { Smith Jr, } \\
2000 .\end{array}$ & I & $\begin{array}{c}\text { Modelo } \\
\text { baseado } \\
\text { em VC }\end{array}$ & Urbana & $\begin{array}{l}\text { Aprimorar o } \\
\text { modelo de } \\
\text { previsão de } \\
\text { demanda de } \\
\text { viagens } \\
\text { rodoviárias }\end{array}$ & $\mathrm{DE}, \mathrm{POP}$ & Rodoviário & $\begin{array}{l}\mathrm{GC}, \mathrm{DC}, \\
\mathrm{CV}, \mathrm{AT}\end{array}$ & 28 setores da economia & $\begin{array}{l}\text { Estado de } \\
\text { Wisconsin }\end{array}$ \\
\hline $\begin{array}{l}\text { Holguín- } \\
\text { Veras e } \\
\text { Thorso, } \\
2000\end{array}$ & I & $\begin{array}{c}\text { Modelo } \\
\text { baseado } \\
\text { em VC }\end{array}$ & $\begin{array}{c}\text { Urbano / } \\
\text { Interurban } \\
\text { o }\end{array}$ & $\begin{array}{c}\text { Comparar os } \\
\text { resultados da } \\
\text { aplicação de } \\
\text { modelos baseados } \\
\text { em VC e em VV. }\end{array}$ & $\begin{array}{l}\text { Unidade, } \\
\text { Peso, VC. }\end{array}$ & Rodoviário & $\begin{array}{c}\text { GC, } \\
\text { DC, ma } \\
\text { CV, AT }\end{array}$ & $\begin{array}{l}\text { Material de construção, } \\
\text { minerais, produtos } \\
\text { aanufaturados, combustíveis, } \\
\text { artigos de metais e têxteis, } \\
\text { alimentos, equipamento } \\
\text { elétrico. }\end{array}$ & $\begin{array}{l}\text { Rodovia da } \\
\text { cidade de } \\
\text { Guatemala }^{5}\end{array}$ \\
\hline
\end{tabular}

Nota: $\mathrm{DE}=$ Dados de empregados, $\mathrm{POP}=$ População, $\mathrm{VC}=$ Volume de carga, VV= Volume de veículos, GC=Geração de carga, DC=Distribuição de carga, $\mathrm{DM}=$ Divisão modal, $\mathrm{CV}=$ Carregamento do veículo, $\mathrm{AT}=\mathrm{Alocação} \mathrm{de} \mathrm{tráfego,} \mathrm{GV}=\mathrm{Geração} \mathrm{de} \mathrm{viagens,} \mathrm{DV=Distribuição} \mathrm{de} \mathrm{viagens.}$

\footnotetext{
${ }^{5}$ Fonte: elaboração própria.
} 
Tabela 1 - Síntese das referências selecionadas sobre aplicação do modelo de quatro etapas - continuação

\begin{tabular}{|c|c|c|c|c|c|c|c|c|c|}
\hline Referência & $\begin{array}{l}\mathrm{Nac} / \\
\text { Intl }\end{array}$ & $\begin{array}{l}\text { Tipo de } \\
\text { fluxo }\end{array}$ & $\begin{array}{l}\text { Abrangência } \\
\text { geográfica }\end{array}$ & Objetivos & $\begin{array}{c}\text { Variáveis } \\
\text { consideradas }\end{array}$ & $\begin{array}{c}\text { Modos } \\
\text { considerados }\end{array}$ & $\begin{array}{c}\text { Etapas } \\
\text { consideradas }\end{array}$ & Produtos considerados & $\begin{array}{l}\text { Local do } \\
\text { estudo }\end{array}$ \\
\hline Black, 1999 & I & $\begin{array}{c}\text { Modelo } \\
\text { baseado } \\
\text { em VC }\end{array}$ & Estadual & $\begin{array}{l}\text { Criar um banco } \\
\text { de dados para } \\
\text { auxiliar a tomada } \\
\text { de decisão e } \\
\text { servir a pesquisas } \\
\text { futuras. }\end{array}$ & TE, POP & $\begin{array}{l}\text { Rodoviário e } \\
\text { Ferroviário }\end{array}$ & $\begin{array}{l}\mathrm{GC}, \mathrm{DC}, \mathrm{DM}, \\
\mathrm{CV}, \mathrm{AT}\end{array}$ & 23 tipos de carga & $\begin{array}{l}\text { Estado de } \\
\text { Indiana }\end{array}$ \\
\hline $\begin{array}{c}\text { CETU, } \\
1999\end{array}$ & I & $\begin{array}{c}\text { Modelo } \\
\text { baseado } \\
\text { em VC }\end{array}$ & Nacional & $\begin{array}{l}\text { Elaborar um } \\
\text { modelo de } \\
\text { previsão de } \\
\text { demanda } \\
\text { aplicável a } \\
\text { estudos urbanos } \\
\text { e interurbanos }\end{array}$ & Não foi informado & $\begin{array}{l}\text { Rodoviário, } \\
\text { Aéreo, } \\
\text { Ferroviário, } \\
\text { Aguaviário, } \\
\text { Dutoviário e } \\
\text { combinações. }\end{array}$ & $\begin{array}{l}\mathrm{GC}, \mathrm{DC}, \mathrm{DM}, \\
\mathrm{CV}, \mathrm{AT}\end{array}$ & $\begin{array}{c}\text { Animais, vegetais, } \\
\text { mineração, vidro } \\
\text { produtos químicos, } \\
\text { borracha e plástico, } \\
\text { madeira, celulose, } \\
\text { papel } \\
\text { têxteis, material de } \\
\text { construção, metal, } \\
\text { máquinas, eletrônicos, } \\
\text { material de transporte, } \\
\text { Outros }\end{array}$ & $\begin{array}{l}\text { Estados } \\
\text { Unidos }\end{array}$ \\
\hline $\begin{array}{l}\text { Souleyrette } \\
\text { et al, } 1998\end{array}$ & I & $\begin{array}{c}\text { Modelo } \\
\text { baseado } \\
\text { em VC }\end{array}$ & Estadual & $\begin{array}{c}\text { Desenvolver um } \\
\text { modelo } \\
\text { simplificado }\end{array}$ & Não foi informado & $\begin{array}{l}\text { Selecionados } \\
\text { conforme } \\
\text { importância } \\
\text { para a região. }\end{array}$ & $\begin{array}{c}\mathrm{GC}, \mathrm{DM}, \mathrm{DC}, \\
\mathrm{AT}\end{array}$ & $\begin{array}{l}\text { Produtos frigoríficos e } \\
\text { maquinário agrícola }\end{array}$ & $\begin{array}{l}\text { Estado de } \\
\text { Iowa }^{6}\end{array}$ \\
\hline
\end{tabular}

Nota: $\mathrm{NE}$ = Número de empregados, ZT= Zona de tráfego, $\mathrm{POP}=$ População, $\mathrm{POPa}=$ População ativa, ND= Número de domicílios, TX=Taxa de emprego, VC= Volume de carga, VV= Volume de veículos, GC=Geração de carga, DC=Distribuição de carga, DM=Divisão modal, CV= Carregamento do veículo, AT=Alocação de tráfego, GV=Geração de viagens, DV= Distribuição de viagens.

\footnotetext{
${ }^{6}$ Fonte: elaboração própria.
} 
Muñuzuri et al. (2009) em um estudo realizado na cidade de Sevilla, apresentam um modelo de planejamento de transporte de carga urbana, com foco no tráfego existente entre atacadistas e distribuidores. Os autores buscaram desenvolver um modelo que melhor se adequasse a realidade urbana e que necessitasse de uma menor quantidade de dados para sua utilização, tornando-se de aplicação mais simples.

Como indicadores para a estimativa de atração e produção de viagens foram utilizados dados relativos à quantidade de atacadistas e distribuidores na região estudada e dados da população. Para a distribuição de viagens foi utilizado o modelo gravitacional suportado pela técnica de maximização da entropia, que, segundo o autor, se adéqua melhor a situações onde as zonas de tráfego são muito próximas. O uso do conceito de maximização da entropia e minimização da informação possui aplicações em redes de transporte e tem como premissa que a matriz OD com maior entropia, que apresenta o arranjo de maior desordem, será também a matriz O-D com maior probabilidade de ocorrer ou de ser observada (LOUREIRO, 2010).

O conceito de entropia introduzido por Shannon (1948) pode ser entendido como entropia na teoria da informação e refere-se à incerteza de uma distribuição de probabilidade. Tem-se três tipos de incerteza: a incerteza determinística, em que os estados que um sistema pode assumir não são conhecidos; a incerteza entrópica, em que os estados possíveis são conhecidos, mas não as chances de ocorrência de cada um deles e a incerteza probabilística, em que os estados possíveis são conhecidos e também a distribuição de probabilidade para eles. Aplicações em transporte do conceito de entropia são encontradas em Wilson $(1967,1970)$, onde a partir deste conceito chegou-se em modelos gravitacionais.

Muñuzuri et al. (2009) abordam apenas o modo rodoviário. A alocação das viagens à rede é realizada através do software EMME $2^{\circledR}$ e os autores destacam que o modelo precisa ser validado.

O modelo apresentado por MT (2007) propõe a retomada do processo de planejamento no setor dos transportes envolvendo todos os modos. O modelo de quatro etapas foi utilizado de modo seqüencial (1- geração de viagens, 2- distribuição de viagens, 3- divisão modal e 4alocação de tráfego). Porém, segundo Marra (1999), o ideal seria tratar as cargas de forma mais detalhada. Entretanto, a desagregação em excesso tende a onerar demasiadamente o 
estudo, podendo inviabilizá-lo. Dessa forma, o trabalho apresenta 13 grupos de produtos considerados relevantes para o estudo.

Para a geração de viagens foi considerada parte da cadeia de suprimento dos grupos de produtos, excetuando-se o grupo de carga geral para o qual foram consideradas matrizes de relacionamento econômico entre as regiões estimadas pelo modelo EFES da FIPE (Fundação Instituto de Pesquisas Econômicas). A distribuição de viagens dos produtos considerados commodities foi realizada com base no modelo gravitacional. Já para os produtos de baixo volume e maior valor agregado considerados como carga geral utilizou-se intercâmbio comercial entre regiões, em valores monetários, fornecidos pelo modelo EFES. Não foi especificado o método utilizado para a realização da divisão modal. A alocação dos fluxos de veículos à rede de transporte foi realizada através de simulação com o uso do software TransCAD e validada por meio de comparação com os dados pesquisados em campo.

Hoeguin-Veras e Thorso (2000) realizaram um estudo da cidade de Guatemala, onde compararam os dados obtidos através de um modelo baseado em volume de carga e um modelo baseado em viagens de veículos. A geração de viagens foi baseada em métodos matemáticos de regressão e a distribuição baseada no modelo gravitacional. Quanto à alocação de tráfego, o autor informa apenas que esta foi realizada segundo as técnicas usuais, descritas no item 2 deste artigo. No caso do modelo baseado em volume de carga, houve a necessidade de converter os volumes obtidos em viagens de veículos para que estas pudessem ser, posteriormente, alocadas a rede de tráfego.

Neste estudo, verificou-se que cada abordagem apresenta vantagens e desvantagens, sugerindo que a integração destas poderia proporcionar melhor compreensão do transporte de carga em uma região, sendo necessário para tanto, um entendimento da relação existente entre a distribuição das distâncias das viagens (DDV) de carga e de veículos. As DDVs, independente, de serem definidas pela abordagem de volume de carga ou de viagens de veículos, apresentaram distribuição assimétrica, com maior concentração em pequenas distâncias, no entanto, tendem a exibir um comportamento consistentes se comparadas.

Sorrati e Smith Jr (2000) apresentaram um estudo realizado no Estado de Wisconsin com o objetivo de aprimorar o modelo de previsão de viagens rodoviárias no âmbito estadual. O modelo apresentado é baseado no volume de carga e utiliza basicamente dados provenientes 
de pesquisas existentes. A produção foi estimada com base em dados da pesquisa de fluxo de carga (commodity flow survey - CFS). Já a atração foi obtida por meio de um modelo econométrico de entradas e saídas de Wisconsin. Ambos os itens foram primeiramente obtidos em valores monetários para, posteriormente, serem convertidos para peso (toneladas).

Para distribuição do volume de carga utilizou-se uma combinação do modelo gravitacional e do modelo Fratar, que é um aprimoramento dos métodos fator de crescimento uniforme e fator médio de crescimento. Por considerar apenas o modo rodoviário, não foi realizada a etapa de divisão modal. Por ser baseado em volume de carga foi necessário converter os valores obtidos para número de viagens. Essa conversão é feita considerando a densidade da carga e a capacidade dos modos. A alocação de fluxos foi realizada por meio do software TRANPLAN.

O estudo apresentado pelo CETU (1999) teve por objetivo desenvolver uma metodologia para previsão de demanda de fluxos de carga que possa ser aplicada a estudos urbanos e interurbanos. $\mathrm{O}$ trabalho apresentado usa principalmente dados secundários obtidos por meio de pesquisas anteriores. Primeiramente ele relaciona os grupos de carga aos setores econômicos e industriais, obtendo a atração e produção para o ano base e estimando-a para o ano de projeto. Para distribuição dos fluxos de carga, o autor recomenda o modelo gravitacional.

Os modos de transporte considerados para divisão modal são diversos, pois o estudo considera, além dos tradicionais (aéreo, rodoviário, ferroviário e hidroviário), uma série de composições entre esses modos. Para realização da divisão modal, o estudo sugere algumas alternativas, como a utilização de tabelas fornecidas pelo CFS, opinião de especialistas e os modelos logit ou pivot point desenvolvidos para divisão modal.

Como o estudo baseia-se em volume de carga, é necessário primeiramente converter o volume de toneladas/ano para toneladas/dia. Posteriormente, o volume em toneladas/dia é convertido para o modo de transporte determinado de acordo com a densidade da carga e a capacidade do modo. Para realizar a alocação de tráfego, o trabalho menciona o método de menor caminho como o mais utilizado, mas informa a existência de outras técnicas. 
O modelo apresentado por Black (1999), sobre o Estado de Indiana, baseia-se no volume de carga. Este estudo teve por objetivo criar um banco de dados que pudesse auxiliar a tomada de decisão e servir para pesquisas futuras. $\mathrm{O}$ autor utilizou métodos matemáticos de regressão para a etapa de geração de viagens e o tradicional modelo gravitacional para a etapa de distribuição de viagens entre as zonas de tráfego.

Foram considerados apenas os modos rodoviário e ferroviário para o estudo. A divisão modal foi realizada com o auxilio de modelo computacional NEWMODE. Os fluxos foram alocados à rede utilizando a técnica do caminho mínimo, porém, sem considerar restrições de capacidade. Posteriormente foi necessário realizar ajustes dos dados obtidos.

O estudo apresentado pelos autores Souleyrette et al. (1998) utilizou uma abordagem do modelo de quatro etapas simplificada, onde primeiramente são definidas as cargas relevantes. Nesse caso foram definidos apenas dois tipos de carga: produtos frigoríficos e maquinário agrícola, por serem essas as mais relevantes para o Estado de Iowa. O restante do processo é realizado por tipo de carga. Os dados para obtenção da atração e produção foram obtidos diretamente de fontes secundárias.

A divisão modal é realizada diretamente após a definição dos tipos de carga, pois cada tipo é alocado ao modo que lhe é mais característico. A distribuição de viagens foi realizada com base no modelo gravitacional por meio do software Map-Info. A alocação de tráfego foi realizada utilizando o software TRANPLAN. Os autores consideram esse projeto como um piloto, no qual foram testados apenas dois tipos de produto, mas explicam que estão trabalhando para expandir o modelo para outras cargas e modos de transporte.

\section{Discussão da revisão bibliográfica}

Considerando todas as 44 referências analisadas é possível verificar que o modelo de análise e previsão de demanda de quatro etapas representa uma ferramenta versátil para apoiar o planejamento de transporte de carga, podendo ser aplicado para diferentes abrangências geográficas (de municípios a países), para diferentes tipos de carga (de carga geral com baixos volumes e altos valores agregados até granéis com elevados volumes e baixos valores 
agregados), considerando o fluxo em unidades de carga ou de veículos e considerando todos os modos de transporte ou suas combinações.

Todos os trabalhos considerados tratam os fluxos de carga de acordo com a sua tipologia, diferenciando a obtenção de informações e dados por tipo de carga. Esta situação destaca a complexidade que pode ocorrer na aplicação no modelo de análise e previsão de demanda de quatro etapas quando se considera uma área de estudo muito abrangente (estados, regiões macro-econômicas, países ou até mesmo continentes), quando um conjunto muito grande de tipos de carga deve ser considerado no planejamento estratégico do transporte de carga. Por outro lado, foi possível encontrar aplicações para os mais diferentes tipos de carga, como pode ser visto na Tabela 1, envolvendo carga geral de diferentes tipos, formas de agregação e valores, inclusive carga conteinerizadas; granéis sólidos (minerais e agrícolas); granéis líquidos e commodities agrícolas. Estas aplicações reforçam a flexibilidade e abrangência de aplicação do modelo de quatro etapas.

Para os 16 estudos que adotam uma visão abrangente para a modelagem do planejamento de transporte de carga, considerando aspectos relacionados ao transporte e ao desenvolvimento socioeconômico o enfoque utilizado é aderente aquele apresentado na Figura 1 e consideram, em maior ou menor grau, aspectos relacionados ao transporte e seu impacto no desenvolvimento socioeconômico por meio do uso de parâmetros e variáveis econométricos e métodos causais. Estes trabalhos possibilitam reflexões sobre a modelagem a ser adotada no planejamento do transporte de carga quando este compõe o planejamento estratégico para o desenvolvimento sócio-econômico de uma região de estudo e neste caso o modelo de quatro etapas é considerado como um elemento central do processo de planejamento.

O conteúdo destes estudos ajuda a entender como o modelo de análise e previsão de demanda de quatro etapas pode ser aplicado ao planejamento estratégico do transporte de carga, indicando quais componentes do sistema de atividades, do sistema de transporte e do contexto institucional devem ser considerados e priorizados na atividade de planejar transportes (Figura 1). Também colaboram com a indicação sobre informações e dados que devem ser coletados (sócio-economia, distribuição de atividades, zoneamento, infraestrutura, rede de transporte entre outros) e a seqüência de aplicação do procedimento de modelagem. Por posicionarem o modelo de análise e previsão de demanda no centro do modelo de planejamento de transportes, estes trabalhos também oferecem sugestões para os relacionamentos entre o 
modelo de quatro etapas e as demais atividades relacionadas ao planejamento estratégico do transporte de carga.

Quando o enfoque é dado a aplicação do modelo de quatro etapas, este foi apresentado e aplicado de forma completa ou parcial, neste caso, enfocando-se uma das quatro etapas. Porém, não se verificou na literatura consultada o enfoque na alocação de fluxo de forma isolada. Esta situação pode estar relacionada a uma deficiência da pesquisa, tendo em vista que estudos de alocação de fluxo podem estar mais associados a engenharia de tráfego, área de pesquisa não considerada, uma vez que a presente pesquisa se concentrou nas áreas de planejamento de transporte de carga.

Os trabalhos que enfocam a geração (atração e produção) de viagens utilizam métodos baseados em séries temporais, métodos causais e taxas para previsão futura. Não se pode afirmar qual destes métodos é mais fácil de aplicar ou apresenta melhores resultados, sendo a sua escolha dependente da disponibilidade de dados confiáveis e do entendimento que se dispõe das características sócio-econômicas e físico-geográficas da região de estudo e suas zonas de tráfego. Há uma predominância no uso de métodos causais, que possibilitam associação entre o comportamento do sistema de transporte de carga com parâmetros e variáveis sócio-econômicos e físico-geográficos das zonas de tráfego, permitindo assim que esta ferramenta de previsão possa ser utilizada de forma mais eficiente no apoio ao desenvolvimento sócio-econômico da região de estudo pela sua inserção nos modelos de planejamento para o desenvolvimento sócio-econômico.

Via de regra, os trabalhos que enfocam a distribuição de viagens utilizam o modelo gravitacional para a determinação dos fluxos de carga interzonais. As variáveis de geração de viagem (atração e produção) são predominantemente consideradas na forma de unidades de carga a serem transportadas e a impedância ou fator de fricção mais utilizado é a distância entre os centróides das zonas de tráfego. Estas variáveis são usualmente escolhidas pela facilidade em sua obtenção (disponibilidade de dados).

A utilização do modelo gravitacional vinculado e de fatores de ajuste para convergência dos resultados, aplicados por meio de técnicas de programação linear, parecem ajudar a melhorar os resultados obtidos (aproximam a previsão dos resultados colhidos em campo, quando os trabalhos apresentaram esta informação). Porém, em previsões de médio e longo prazos (para 
horizontes superiores a 5 anos) a precisão sempre tende a diminuir.

Os trabalhos que apresentam o método de maximização de entropia aplicado a distribuição de viagens demonstram que este método ajuda a melhorar a previsão dos resultados de aplicação do modelo gravitacional. Por outro lado, a programação linear na forma do problema de transporte têm aplicação limitada em abrangência geográfica e diversidade de cargas.

Quando o enfoque é dado a divisão modal, predomina a técnica de modelagem convencional, com uso de modelos LOGIT binomial e multinomial. Porém se observou o uso do modelo PROBIT e até a aplicação de lógica fuzzy utilizando como parâmetros o custo (de transporte ou generalizado), o tempo de viagem e o nível de serviço.

A maioria dos estudos verificados baseia-se em volume de carga transportada, aderindo ao procedimento apresentado na Figura 3. Isso ocorre por que esse procedimento permite captar detalhes sobre os atributos necessários à avaliação das variáveis que influenciam a movimentação das cargas. Nesse procedimento, os produtos relevantes são definidos antes de iniciar o modelo de quatro etapas. Excetuando-se o estudo de MT (2007), que menciona ter verificado parcialmente a cadeia produtiva das cargas (cadeia de suprimento), os demais estudos não deixam isso claro.

Quando o foco do estudo encontra-se nas viagens de veículo (Figura 2), a coleta de dados torna-se mais simples, devido aos controles de tráfego existentes atualmente. No entanto, usualmente não apresentam detalhes sobre a carga transportada.

Modelos de previsão de demanda são complexos, devido, principalmente, a quantidade de dados necessária a sua aplicação. Verificou-se que nos Estados Unidos existem diversos bancos de dados, resultantes de pesquisas realizadas pelo governo e outros órgãos, com o intuito de disponibilizar informações para planejamento estratégico, tornando esse planejamento mais simples em sua aplicação. Os estudos realizados nos Estados de Indiana, Iowa e Wisconsin (Black, 1999 e Sorratini e Smith Jr, 2000), utilizaram amplamente dados secundários para aplicação de seus modelos.

Devido sua abrangência, estudos com abordagem nacional são de difícil implementação. Neste sentido, foram analisadas duas propostas: MT (2007) e CETU (1999). Ambos os estudos consideram diversos modos de transporte e grupos de carga e possuem etapas 
similares, resguardando suas particularidades. Por possuir diversos dados disponíveis, o CETU (1999) orienta para que esses dados sejam utilizados na aplicação do modelo, tornando-o mais simples e viável. No caso do MT (2007), o Brasil ainda carece de uma melhor estrutura que auxilie tais planejamentos. Sendo assim, o estudo necessitou de intensiva coleta de dados.

Grande parte dos trabalhos restringiu-se ao perímetro urbano e, por conseqüência, ao modo rodoviário. Esses estudos apresentaram uma estrutura simplificada do modelo de quatro etapas (Figura 2), pois ao restringirem o uso ao modo rodoviário, eliminaram a etapa de divisão modal, simplificando sua aplicação.

De forma geral, o modelo de quatro etapas parece adequar-se melhor ao planejamento do transporte de carga de forma agregada, quando se deseja estabelecer tendências de fluxo de carga e comportamentos comuns a diferentes setores de produção. Com isso, representa uma ferramenta útil para apoiar o planejamento do crescimento econômico de uma região e contribuir para a melhoria da qualidade de vida da sociedade.

Por outro lado, o modelo de quatro etapas quando aplicado a análise e previsão de demanda de fluxos de carga não parece ser capaz de modelar diretamente os aspectos relacionados ao planejamento estratégico do transporte de carga como função logística, representando uma aparente limitação da técnica.

Esta limitação decorre do fato de que para a aplicação deste modelo não é necessária a especificação da cadeia de suprimento para um determinado tipo de carga. Esta situação pode levar a distribuição inadequada de fluxos (Tij) por ZTs que não pertencem a uma cadeia de suprimento específica, mas que, por outro lado, atende às restrições impostas pelo modelo gravitacional. Ao aplicá-lo, este carrega consigo todas as aproximações e ajustes da etapa de geração de viagens e propaga as imprecisões para a etapa de divisão modal. O resultado será adequado ao modelo, mas pode ser inadequado à realidade observada. Naturalmente, existem métodos de calibração dos resultados obtidos, mas deve-se avaliar até que ponto não se estará introduzindo um excesso de artificialidade no modelo.

Adicionalmente, as propostas e aplicações do modelo de quatro etapas aqui apresentadas destinam-se especificamente a análise e previsão de demanda de fluxos de transporte, 
considerados de forma isolada. Estas aplicações, até onde se pode observar, não consideram um enfoque logístico, onde outras funções, como gestão de estoque e processamento de pedido, também contribuem para a configuração de uma cadeia de suprimento, dado um patamar de custo e um nível de serviço.

\section{Conclusão}

Este trabalho preencheu uma lacuna na geração e divulgação de conhecimento sobre gestão de transporte de carga, uma vez que por meio de revisão bibliográfica não exaustiva no período de 1995 a 2010, selecionou 44 trabalhos que tratam do planejamento de transporte de carga, destacando para análise mais detalhada 7 trabalhos que enfocaram na aplicação do modelo de análise e previsão de demanda de quatro etapas, seu objetivo principal. Os resultados desta análise permitem identificar a flexibilidade e abrangência desta ferramenta de planejamento e seu universo de aplicação, destacando a sua potencialidade em apoiar o planejamento sócioeconômico de uma região sob o enfoque estratégico.

A revisão bibliográfica realizada identificou uma pequena participação de referências nacionais (9\%) sobre o tema, o que ratifica a percepção de existência de lacuna na publicação de trabalhos sobre o uso do modelo de quatro etapas como ferramenta de planejamento de transporte de cargas no Brasil em periódicos científicos. Não foi possível identificar se isso se deve a pequena difusão do uso deste modelo para esta aplicação (planejamento do transporte de carga) ou pela pouca ênfase sobre esta ferramenta em cursos de formação profissional na área de transportes com enfoque no transporte de carga. De qualquer forma, embora não tenha sido o foco do presente trabalho, outras pesquisas dos mesmos autores deste trabalho identificaram um número maior de publicações nacionais sobre o uso do modelo de quatro etapas para o planejamento do transporte de passageiros.

Com isso espera-se contribuir com a difusão do potencial de utilização deste modelo de planejamento de transportes, o que pode ser de aplicação muito útil para o desenvolvimento do planejamento do transporte de carga no Brasil, apoiando seu desenvolvimento socioeconômico, tendo em vista a importância do transporte como indutor de desenvolvimento. 
Pelo que se pôde verificar, o modelo de análise e previsão de demanda de quatro etapas representa uma ferramenta versátil para apoiar o planejamento de transporte de carga, podendo ser aplicado para diferentes abrangências geográficas, para diferentes tipos de carga, considerando o fluxo em unidades de carga ou de veículos e considerando todos os modos de transporte ou suas combinações. Assim sendo, entende-se que o modelo de quatro etapas se adéquie ao planejamento estratégico do transporte de carga sob a ótica predominantemente adotada pelo poder público, onde na maioria das aplicações não se considera a configuração da cadeia de suprimento das cargas estudadas.

Por outro lado, o enfoque dos embarcadores e transportadores não parece ser considerado neste modelo de planejamento, o que pode levar a um descasamento entre as necessidades da iniciativa privada, que embarca e movimenta as cargas e considera uma visão segmentada do setor econômico onde atua e o planejado pelo poder público, usualmente o supridor de infraestrutura para esta atividade.

Neste sentido, entende-se que o modelo poderia ser aprimorado se considerado o enfoque logístico em sua formulação e aplicação, de modo que se pudesse adaptá-lo como uma ferramenta de planejamento logístico, onde não apenas a função transporte fosse modelada, mas também as funções gestão de estoque e processamento de pedido. Este aprimoramento implicaria em um melhor entendimento dos fluxos de materiais e produtos finais, considerando o fluxo logístico (fluxo de transporte, acúmulos de estoque e troca de informações) e não apenas o fluxo de transporte.

A revisão bibliográfica realizada permitiu identificar trabalhos que consideram a aplicação do modelo de quatro etapas, em sua totalidade ou de forma parcial, como ferramenta de planejamento de transporte de carga. A diversidade de aplicações quanto a abrangência geográfica, consideração de diferentes tipos de carga, modos de transporte (e suas combinações), locais de aplicação e horizontes de estudo demonstram a abrangência, versatilidade e flexibilidade desta ferramenta. Estas considerações por si só já demonstram a importância desta ferramenta de planejamento de transportes. Porém, os resultados encontrados e discutidos limitam-se a abrangência deste estudo. Entende-se que seja possível aprimorá-los por meio de uma revisão bibliográfica mais abrangente. 


\section{Referências}

Abdelwahab, W. M. (1998) Elasticities of mode choice probabilities and market elasticities of demand: evidence from a simultaneous mode choice/shipment-size freight transport model. Transportation Research Part E, vol. 34, n. 4, pp. 257-266.

Adler, H. A. (1978) Avaliação econômica dos projetos de transporte: metodologia e exemplos. Livros Técnicos e Científicos, RJ.

Agência Nacional de Transportes Terrestres - ANTT (2004) Logística e transporte para produtos de alto valor agregado no contexto brasileiro. Relatório Executivo. Brasília, DF.

Al-Deek, H., Klodzinski, J., El-Helw, A., Sarvareddy, P. e Emman, E. (2002) Development of a statewide model for heavy truck freight movement on external networks connecting with Florida ports. Technical Report. Florida, USA.

Allen, W. G. (2002) Development of truck models. Baltimore Metropolitan Council.

Anderson, M. D. (1994) Manual of transportation engineering studies. Englewood Cliffs: Prentice Hall.

Arnold, P., Peeters, D. e Thomas, I. (2004) Modelling a rail/road intermodal transportation system. Transportation Research Part E, vol. 40, pp. 255-270.

Ballou, R. H. (2001) Gerenciamento da Cadeia de Suprimentos: Planejamento, Organização e Logística Empresarial. Trad. Elias Pereira, 4a. ed. Porto Alegre: Bookman.

Beuthe, M., Jourquina, B. Geertsc, J. F., Ha, C. K. (2001) Freight transportation demand elasticities: a geographic multimodal transportation network analysis. Transportation Research Part E, vol. 37, n. 4 , pp. 253-266.

Black, W. R. (1999) Commodity flow modeling. Transportation Research Board, n. E-C011.

Broga, J. J., Brich, S. C. e Demetsky, M. J. (2001) Application of a statewide intermodal freight planning methodology. Virginia Center for Transportation Innovation \& Research Report.

California Environmental Protection Agency e Business - CEPA (2007) Goods movement action plan. Transportation and Housing Agency Goods.

Caris, A., Macharis, C. e Janssens, G., K. (2008) Planning problems in intermodal freight transport: accomplishments and prospects - Transportation Planning and Technology, vol. 31, pp. 277302.

Celik, H. M. e Guldmann, J-M. (2002) Spatial interaction modeling of interregional commodity flows. 42nd European Congress of the Regional Science Association, Dortmund.

Center for Urban Transportation Studies - CETU (1999) Guidebook on statewide travel forecasting. University of Wisconsin - Milwaukee.

Combes, F. e Leurent, F. (2007) Advances in freight transport demand modeling: an assessment with research perspectives, European Transport Conference.

Crainic, P. e Laporte, G. (1997) Planning models for freight transportation. European Journal of Operational Research, vol. 97, n. 3, pp.409-438.

D’Este, G. (1996) As event-based approach to modeling intermodal freight system. International Journal of Physical Distribution \& Logistics Management, vol. 26, n. 6, pp. 4-15.

Federal Highway Administration - FHA, (1999) Guidebook on statewide travel forecasting. Center for Urban Transportation Studies, University of Wisconsin, Milwaukee. 
Garrido, R. A. e Mahmassani, H. S. (1999) Forecasting freight transportation demand with the spacetime multinomial probit model - Transportation Research Part B, vol. 34, pp.403-418.

Harker, P. T. (1985) The state of art in the predictive analysis of freight transport system. Transport Reviews, vol. 5, pp. 143-164.

Hoeguin-Veras, J., Thorson, E. (2000) Trip length distributions in commodity-based and trip-based freight demand modeling- investigations of relationships. Transportation Research Board, $\mathrm{n}$. 1707, pp. 37-48.

Ichihara, S. M. (2007) O impacto do crescimento econômico sobre as rodovias de São Paulo: uma aplicação do modelo de insumo-produto combinado ao geoprocessamento. Revista Economia, vol. 8, n. 4, pp. 199-231.

Jackson, R. W., Schwarm, W. R., Okuyama, Y., Islam, S. (2004) A method for constructing commodity by industry flow matrices - 2004 Southern Regional Science Association Conference, New Orleans

Janic, M. (2007) Modelling the full costs of an intermodal and road freight transport network. Transportation Research Part D, vol. 12, n. 1, pp. 33-44.

Jiang, F., Johnson, P. e Calzada, C. (1999) Freight demand characteristics and mode choice: an analysis of the results of modeling with disaggregate revealed preference data. Journal of Transportation and Statistics, vol. 2, n. 2, pp. 149-158.

Jong, G., Gunn, H. e Ben-Akiva, M. (2004) A meta-model for passenger and freight transport in Europe. Transport Policy, vol. 11, pp. 324-344.

Kanashiro, K. (2003) Um novo olhar para o planejamento de transportes no Brasil. Sindicato dos Terminais Marítimos do Rio Grande do Sul. Disponível em www.sintermar.com.br.

Kawamura, K., Chandler, D., West, N. e Benedict, A. (2006) Development and testing of model planning process for freight oriented development. Urban Planning International, vol. 4.

Kenneth, E. P., Johnson, T., Gilles, J., Fulcher, C., Wilson-Orndoff, C. (2003) Benefits of highway improvements on rural communities in Missouri: economic development considerations Missouri Department of Transportation - Technical Report.

Kveiborg, O. e Fosgerau, M. (2006) Decomposing the decoupling of Danish road freight traffic growth and economic growth. Transport Policy, vol. 14, pp. 39-48.

Loureiro, K. D. (2010). Uso de contagens volumétricas na estimativa de matrizes de origem-destino de veículos leves em redes interurbanas. Tese (Doutorado) - Universidade Federal de Santa Catarina, Florianópolis.

Macharis, C. e Bontekoning, Y. M. (2004). Opportunities for OR in intermodal freight transport research: a review. European Journal of Operational Research, vol. 153, pp. 400-416.

Magalhães, S. L. M. (2008) Planejamento de transporte de um aglomerado urbano com intermodalidade por meio de transportes integrados. Tese (Doutorado em Engenharia de Transportes), Universidade Federal do Rio de Janeiro, Rio de Janeiro.

Makridakis, S., Wheelwrigt, S. e Hyndman, R. J. Forecasting Methods and Applications, 3a ed. Hoboken: Wiley.

Malchow, M. B. e Kanafani, A. (2004) A disaggregate analysis of port selection. Transportation Research Part E, vol. 40, pp. 317-337.

Marins, F. A. S. (2011) Técnicas de previsão de demanda. Material de aula. Universidade Estadual Paulista. 
Marra, C. (1999) Caracterização de demanda de movimentações urbanas de carga. Dissertação de Mestrado em Engenharia Civil, Universidade Estadual de Campinas, SP.

Menezes, U. R. (1971) Introdução ao planejamento de transportes urbano. Recife.

Miller, J. S., Hoel, L. A., Dalton, F. T. e Mccray, D. (2008) Linking transportation planning to program implementation. Journal of Urban Planning and Development, vol. 134, n. 2, pp. 8895.

Ministério dos Transportes (2007) Plano nacional de logística e transporte. Brasil.

Morlok, E. K. (1978) Introduction to transportation engineering and planning. McGraw-Hill, US.

Muñuzuri, J., Cortés, P., Onieva, L. e Guadix, J. (2009) Modeling freight delivery flows: the missing link of urban transport analysis. Journal of Urban Planning and Development. Vol. 135, n. 3, pp. 91-99.

Ogden, K. W. (1977) Modeling urban freight generation . Traffic Engineering \& Control. vol. 18, pp. 106-109.

Ogden, K. W. (1992) Urban goods movement: a guide to policy and planning. Ashgate Publishing Limited, London.

Oliveira, R., L. M. e Cury, M., V., Q. (2004) A escolha modal no transporte de cargas sob a ótica da modelagem Neuro-Fuzzy: um estudo de caso. XVIII Congresso de Pesquisa e Ensino em Transportes.

Patterson, Z., Ewing, G. O. e Haider, M. (2008) The potential for premium-intermodal services to reduce freight $\mathrm{CO} 2$ emissions in the Quebec City-Windsor Corridor. Transportation Research Part D, vol 13, pp. 1-9

Perry, E. (2003) Transportation and economic development: which comes first? Supplemental Material for RDT Report, n. 03-013

Regan, A. C. e Garrido, R. A. (2002) Modeling freight demand and shipper behavior: state of the art, future directions. In: Hensher, D. e King, J. (eds.) The Leading Edge of Travel Behavior Research, Oxford: Pergamon.

Ricci, A. e Black, I. (2005) The social cost of intermodal freight transport. Research in Transportation Economics, vol. 14, pp. 245-285.

Shannon, C. E. (1948). A mathematical theory of communication. Bell System Technical Journal, vol. 27, pp. 379-423.

Sorrati J. A. e Smith, R. L. (2000) Development of a statewide truck trip forecasting model based on commodity flows and input-output coefficients. Transportation Research Board, n. 1707.

Souleyrette, R., Maze, T. H., Strauss, T., Preissig, D., Smadi, A. G. (1998) Freight planning typology. Transportation Research Board, n. 1613.

Southwestern Pennsylvania Commission - SPC (2002) The southwestern Pennsylvania freight transport guidebook, Pennsylvania, USA.

Swedish Model System for Planing - SAMPLAN (2001) The Swedish model system for goods transportation - Samgods. A brief introductory overview, SAMPLAN Rapport, n. 2000:1.

Tavasszy L. A., van de Vlist, M. J. M., van Haselen e H. W. J., van der Rest, H. (1997) Freight transportation system modelling: chains, chains and chains. European Transport Conference.

Tedesco, G. M. I. (2008) Metodologia para elaboração do diagnóstico de um sistema de transporte. Dissertação (Mestrado) - Universidade de Brasília, Brasília. 
Villalobos, J. R., Maltz, A., Ahumada, O., Treviño, G., Sánches, O. e Garcia, H. C. (2006) Logistics capacity study of the guaymas-tucson corridor. A Report to the Arizona Department of Transportation.

Wilson, A. G. (1967). A statistical theory of spacial distribution models. Transportation Research, pp. 253-269.

Wilson, A. G. (1970). Entropy in urban and regional modelling. London: Pion. 